\title{
$S$-adenosylmethionine and $S$-adenosylhomocysteine levels in the aging brain of APP/PS1 Alzheimer mice
}

\author{
Carlijn R. Hooijmans · Henk J. Blom • \\ Dinny Oppenraaij-Emmerzaal · \\ Merel Ritskes-Hoitinga · Amanda J. Kiliaan
}

Received: 13 October 2008/ Accepted: 15 June 2009/Published online: 30 June 2009

(C) The Author(s) 2009. This article is published with open access at Springerlink.com

\begin{abstract}
Hyperhomocysteinemia and factors of homocysteine metabolism, $S$-adenosylhomocysteine (AdoHcy) and $S$-adenosylmethionine (AdoMet), may play a role in Alzheimer's disease (AD). With liquid-chromatographytandem-mass-spectrometry AdoMet and AdoHcy were determined in brains of 8- and 15-month-old APP/PS1 Alzheimer mice, and their possible roles in AD brains investigated. The finding that AdoMet levels do not differ between the genotypes in (young) 8-month-old mice, but are different in (older) 15-month-old APP/PS1 mice compared to their wild-type littermates, suggests that alterations in AdoMet are a consequence of AD pathology rather than a cause. During aging, AdoMet levels decreased in the brains of wild-type mice, whereas AdoHcy levels diminished in both wild type and APP/PS1 mice. The finding that AdoMet levels in APP/PS1 mice are not decreased during aging (in contrast to wild-type mice), is probably related to less demand due to neurodegeneration. No effect of the omega-3 fatty acid docosahexaenoic acid (DHA) or
\end{abstract}

C. R. Hooijmans · A. J. Kiliaan $(\bowtie)$

Department of Anatomy and Department of Cognitive

Neuroscience, Donders Centre for Neuroscience,

Radboud University Nijmegen Medical Centre,

6500 HB Nijmegen, The Netherlands

e-mail: A.Kiliaan@anat.umcn.nl

H. J. Blom · D. Oppenraaij-Emmerzaal

Laboratory of Pediatrics and Neurology,

Radboud University Nijmegen Medical Centre,

6500 HB Nijmegen, The Netherlands

M. Ritskes-Hoitinga

Central Animal Laboratory, Radboud University Nijmegen

Medical Centre, 6500 HB Nijmegen, The Netherlands cholesterol-enriched diets on AdoMet or AdoHcy levels were found.

Keywords $S$-adenosylmethionine .

$S$-adenosylhomocysteine · Alzheimer's disease .

APP/PS1 mice $\cdot$ Cholesterol $\cdot$ DHA

\section{Introduction}

The most pronounced pathological features in the human Alzheimer (AD) brain are amyloid- $\beta(\mathrm{A} \beta)$ depositions, intracellular tangles, and neurodegeneration. Although the cause of the disease is largely unknown, the $\mathrm{A} \beta$ protein is seen as one of the major contributors [31].

$\mathrm{A} \beta$ is produced by cleavage of the amyloid precursor protein (APP) by the $\beta$ and $\gamma$ secretases, BACE, and Presenilin (PS). Missense mutations in either APP or the $\gamma$-secretase complex (PS1 and PS2), cause overproduction of $\mathrm{A} \beta$, and early onset $\mathrm{AD}$. Early onset $\mathrm{AD}$ represents only $5 \%$ of all $\mathrm{AD}$ cases and therefore explains only a small part of the cause of the disease. In addition, many studies show that $\mathrm{A} \beta$ depositions do not correlate well with neuronal damage and cognitive decline. Therefore, it is suggested that beside $\mathrm{A} \beta$, other risk factors play an important role in the development of AD. Nowadays, more and more consensus is reached about vascular disorders being major risk factors for AD. Hyperhomocysteinemia for example, was until recently believed to be a risk factor for cardiovascular disorders [23] and associated with an increased risk of AD $[20,28]$. It could therefore be suggested that hyperhomocysteinemia, cardiovascular disorders, and $\mathrm{AD}$ are interrelated with each other. However, this is currently under debate because of recent homocysteine-lowering intervention studies, showing no relation between cardiovascular 
disease and hyperhomocysteinemia [4, 19]. In addition, it is unclear whether lowering of homocysteine (Hcy) by folic acid intake, can diminish AD prevalence or improve cognition [5, 21], because recent large randomized Hcy lowering intervention trials did not show beneficial effect of lowering Hcy with folate and vitamin B12 on cognitive function [21]. Among others, we therefore suggest that hyperhomocysteinemia itself is not causing $\mathrm{AD}$, and that other factors of the Hcy metabolism, such as $S$-adenosylhomocysteine (AdoHcy) and $S$-adenosylmethionine (AdoMet), probably play a more important role in vascular disease [17] and AD development [2, 24, 32].

AdoMet is a biological methyl donor and is a product of the conversion of methionine and ATP. Transfer of the methylgroup from AdoMet leads to the formation of AdoHcy which in turn inhibits cellular methylation. Methylation is required in many essential biological processes, such as gene expression, regulation of protein function, and neurotransmitter synthesis, and altered methylation patterns might play a role in AD [2, 24, 32]. Subsequently, AdoHcy can be hydrolyzed to Hcy and adenosine by AdoHcy hydrolase. However, it is important to mention that this hydrolysis is a reversible reaction that favors the synthesis of AdoHcy. In vivo, the reaction proceeds in the direction of hydrolysis only if the products, adenosine and Hcy, are removed rapidly [6, 18, 22].

Indeed, there are studies showing a role for AdoMet and AdoHcy in $\mathrm{AD}[2,24,30]$. It is suggested that DNA methylation for example, is involved in APP processing and $\mathrm{A} \beta$ production through the regulation of Presenilin 1 (PS1) expression. For example, AdoMet administration in human neuroblastoma cells down regulates PS1 gene expression and $\mathrm{A} \beta$ production [30]. Other studies from the same group showed that alteration in AdoMet/Hcy cycle (due to reduction of folate and vitamin B12 [10] or deprivation of folate and vitamin $\mathrm{B} 12$ and $\mathrm{B} 6[8,9])$ deregulates $\beta$ and $\gamma$-secretases expression and $\mathrm{A} \beta$ production in both neuroblastoma cells and TgCRND8 Alzheimer mice [8-10].

Other studies have shown decreased AdoMet and AdoHcy levels in the cerebrospinal fluid (CSF) of AD patients compared to age-matched healthy volunteers [2, 24].

Until now, most studies determined AdoMet and AdoHcy levels in CSF of AD patients [3]. However, AdoMet and AdoHcy remain mostly intracellular and do not pass membranes easily, and it could therefore be possible that AdoMet and AdoHcy levels in brain tissue comprise a more reliable reflection of $\mathrm{AD}$ processes than $\mathrm{CSF}$ or serum levels which are a 100-fold lower.

There is only one study describing severely decreased AdoMet levels in brain tissue of AD patients [24]. Although the authors carefully matched with respect to postmortem time the results of this study might be difficult to interpret, since AdoMet levels decrease with more than $60 \%$ and AdoHcy levels increase with $80 \%$ within $15 \mathrm{~h}$ after death [25]. In addition, it is still unknown whether alterations in AdoMet and AdoHcy levels are cause or consequence of AD pathology.

In order to further investigate the role of AdoMet and AdoHcy in AD development we determined AdoMet and AdoHcy levels in brain tissue of 8- and 15-month-old wild type and APP/PS1 double transgenic Alzheimer mice. These two ages were chosen because in young (8-monthold) mice no neurodegeneration, almost no cognitive impairment, and very low levels of $\mathrm{A} \beta$ are present, whereas in old APP/PS1 mice (15-month-old) neurodegeneration in the hippocampus becomes clear, and cognitive impairment and $\mathrm{A} \beta$ deposition are quite severe [16].

In addition, we investigated the effect of aging and the effects of cholesterol- and the omega- 3 fatty acid docosahexanoic acid (DHA) containing diets on AdoMet and AdoHcy levels. These nutritional components influence vascular health $[11,13,14,26]$ and the risk of developing $\mathrm{AD}[7,15,29]$. In our previous studies we showed that long-term dietary interventions with cholesterol and or DHA also changed AD-like pathology and vascular factors in 8-and 15-month-old APP/PS1 mice. AdoMet and AdoHcy might also influence AD like pathology and vascular health [16] and it could be hypothesized that the abovementioned nutritional components may influence and AdoMet and AdoHcy levels as well.

\section{Materials and methods}

Animals and diets

The APPswe/PS1dE9 founders were obtained from Johns Hopkins University, Baltimore, MD, USA (D. Borchelt and J. Jankowsky, Dept. of Pathology), and a breeding colony was established at the Radboud University Nijmegen Medical Centre, The Netherlands. Male APP/PS1 transgenic mice and their wild-type littermates were randomly assigned to different diet groups. The number of animals per group was decided on the basis of availability. The experimental diets differed with respect to the composition of the $5 \%$ fat in the diets [15] but are all isocaloric. Feeding the diets started at 2 months of age.

Transgenic mice and their wild-type littermates were fed either a Typical Western Diet (TWD), containing 1\% cholesterol, a high percentage of saturated fatty acids $(\mathrm{SFA}=53 \%)$, and long chain poly unsaturated fatty acid (LCPUFA) ratio of $n 6 / n 3=22.5$, or a DHA diet containing $0.4 \%$ DHA, a low percentage SFA (20\%), and a LCPUFA ratio of $n 6 / n 3=2.5$, or a standard control diet (STD), with $38 \%$ SFA and LCPUFA ratio of $n 6 / n 3=7.5$. 
In total 82 mice were used; Table 1 describes the number of mice used in each experiment. The mice were selected upon availability from another existing study performed in our own lab (submitted). Throughout the experiments the animals were housed individually in a controlled environment (the mice were housed in the central animal facility with the temperature controlled at $21^{\circ} \mathrm{C}$, and an artificial 12:12 h light:dark cycle (lights on at 07.00 am)), with some cage enrichment, consisting of an Iglo and some nesting material. Food and water were available ad libitum. The experiments were performed according to Dutch regulations of the Animal Experimentation Act and the EC Directive 86/609 and were ethically approved by the Ethical Review Committee of the Radboud University Nijmegen Medical Centre.

Brain sample collection and storage

Directly following anesthesia with Nembutal $(60 \mathrm{mg} / \mathrm{kg}$, i.p.) (Ceva Santa Animals BV, Maassluis), all mice were weighed and thereafter decapitated and the brains were removed from the skull. The entire brain, without the spinal cord was thereafter dissected into three smaller pieces, of which the frontal part of the brain was used for this experiment. All pieces were snap-frozen in liquid nitrogen and were kept frozen at $-80^{\circ} \mathrm{C}$ until further processing.

A 40-mg tissue piece, containing the frontal part of the brain was prepared by sonification in $800 \mu \mathrm{l}$ cold PBS, and thereafter centrifuged at $4^{\circ} \mathrm{C}$ at $14,000 \mathrm{rpm}$ for $5 \mathrm{~min}$. The supernatant was removed and diluted eight times. One part was used for HPLC tandem MS measurements, and another part for determination of the protein amount in the sample using the Lowry method [27].

$S$-adenosylmethionine (AdoMet)

and $S$-adenosylhomocysteine (AdoHcy) measurements

As previously described by Gellekink et al. [12], AdoMet and AdoHcy levels in brain tissue were measured using

Table 1 Number of male mice used in each diet group

\begin{tabular}{lll}
\hline Groups & 8 -month-old $(n)$ & 15-month-old $(n)$ \\
\hline STD (wt) & 5 & 10 \\
STD (tg) & 6 & 8 \\
TWD (wt) & 6 & 9 \\
TWD (tg) & 6 & 8 \\
DHA (wt) & 5 & 9 \\
DHA (tg) & 7 & 3
\end{tabular}

STD standard diet, TWD Typical Western Diet, containing 1\% cholesterol, $D H A$ docosahexanoic acid diet, containing $0.4 \%$ of DHA. Wt wild-type mice, $T g$ transgenic APP/PS1 mice liquid chromatography tandem mass spectrometry (LCMS/MS).

In short, after addition of the internal standards AdoMet$\mathrm{d}_{3}$ and AdoHcy- $\mathrm{d}_{5}$ to the pretreated tissue sample (see above), solid phase extraction (SPE) columns containing phenyl boronic acid (PBA) were used to bind AdoMet and AdoHcy and their internal standards and to deproteinize the samples. AdoMet and AdoHcy were eluted in $0.1 \mathrm{~N}$ formic acid and measured with the LC tandem mass spectrometer (MS/MS) (Quatro LC from Micromass), in the positive-ion mode.

\section{Statistics}

Linear regression analysis was used to verify the linearity of the calibration curves. In order to analyze the possible differences between the two genotypes and the different diet groups a Multivariate ANOVA was conducted with the between group factors diet and genotype. In order to investigate effects of aging in the two different genotypes a Multivariate ANOVA was used with the between group factors age and genotype.

\section{Results}

No changes in bodyweight were found between the genotypes, the two different age groups or diet groups.

\section{Genotype effects}

No changes in AdoMet and AdoHcy levels, and consequently in the ratio between AdoMet and AdoHcy levels (methylation index), were observed between 8-month-old wild type and APP/PS1 mice (Fig. 1a-c). In addition, also in 15-month-old mice no changes were observed in AdoHcy levels between the genotypes (Fig. 1b). However, we did observe a significant decrease in AdoMet levels in 15month-old wild-type mice compared to APP/PS1 mice (Fig. 1a; $F(1,45)=7.54, p<0.01$ ). Consequently, the AdoMet/AdoHcy ratio is increased in the APP/PS1 mice (Fig. 1c; $((1,45)=7.17, p<0.01)$.

\section{Aging effects}

AdoHcy levels significantly decrease during aging in both wild-type and APP/PS1 mice $(F(1,79)=9.50, p<0.01)$. Due to an interaction between genotype and age in AdoMet levels and the ratio between AdoMet and AdoHcy levels (methylation index), the effects of aging in wild type and APP/PS1 mice were determined separately for these parameters. 

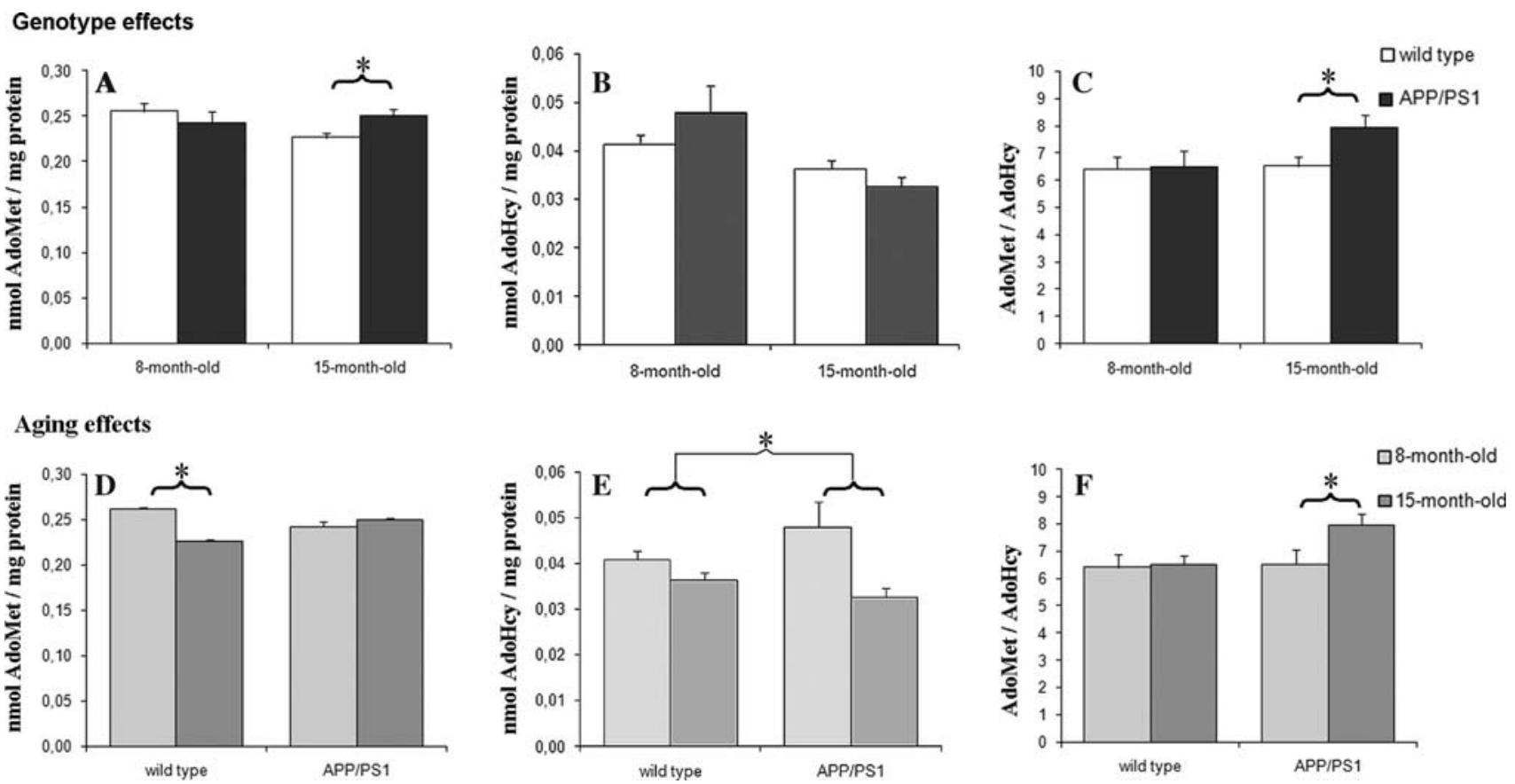

Fig. 1 Levels of $S$-adensylmethionine (AdoMet; a, d) and $S$-adenosylhomocysteine (AdoHcy; b, e) and the methylation index (AdoMet/ AdoHcy; $\mathbf{c}, \mathbf{f}$ ) in brain tissue of 8- and 15-month-old APP/PS1 mice and their wild-type littermates. 8-month-old wild-type mice $n=16$,

8-month-old APP/PS1 mice $n=19$ 15-month-old wild-type mice $n=28,15$-month-old APP/PS1 mice $n=19$. Values represent mean and SEM. $* p<0.01$

A significant decrease in AdoMet levels during aging was only found in wild-type mice $(F(1,42)=8.09$, $p<0.01)$ and not in APP/PS1 mice.

Thus, AdoHcy decreased in both wild type and APP/PS1 mice during aging, whereas AdoMet only decreased in wild-type mice. Consequently, the AdoMet/AdoHcy ratio (methylation index) in wild-type mice did not alter due to aging, but was significantly increased in APP/PS1 mice with aging (Fig. 1f; $F(1,36)=6.61, p=0.01$ ).

Diet effects

Neither a cholesterol- nor a DHA-containing diet did alter AdoMet or AdoHcy levels in brain tissue of 8- and 15month-old APP/PS1 and wild-type mice as compared to the standard diet (data not shown).

\section{Discussion}

In this study we showed that AdoMet tissue levels did not differ between 8-month-old APP/PS1 mice and wild-type littermates, but are decreased in 15-month-old wild-type mice compared to APP/PS1 mice. Moreover, tissue levels of AdoHcy decreased in both wild type and APP/PS1 mice during aging, whereas AdoMet only decreases in wild-type mice. Because AdoMet and AdoHcy brain levels in

(young) 8-month-old mice are unchanged, in contrast to the (old) 15-month-old mice, these results indicate that alterations in AdoMet may be a consequence of AD-like pathology in the APP/PS1 mice rather than a cause. In addition, we showed that cholesterol-containing, or DHAenriched diets did not affect AdoMet or AdoHcy levels in brain tissue of APP/PS1 and wild-type mice.

This is the first study describing AdoMet and AdoHcy levels in brain tissue of aging mice. Stramentinoli et al. found decreased AdoMet levels in brain tissue of aging rats [33]. In addition they measured the synthesis of AdoMet by adenosyl transferase and AdoMet utilization by COMT, and concluded that the decrease in AdoMet levels was a consequence of increased utilization during aging. The decrease in AdoMet and AdoHcy found in our wild-type mice could also be the consequence of increased utilization. It could be suggested that AdoMet levels in the brain tissue of the APP/PS1 mice, used in this study, do not decrease during aging because of less demand caused by neurodegeneration. This seems to be confirmed by the hippocampal atrophy and increased $\mathrm{A} \beta$ deposition we observed in the hippocampus and frontal cortex of the 15month-old APP/PS1 mice compared to 8-month-old APP/ PS1 mice [16]. If the above-mentioned hypothesis is valid, and AdoMet remains unaltered in aged APP/PS1 mice compared to young APP/PS1 mice, because of less demand for methylation due to neurodegeneration, AdoHcy levels 
will also be expected to be decreased or unchanged. Indeed, AdoHcy levels are similar in the 15-month-old wild-type mice and APP/PS1 mice. In Fig. 2, the abovementioned findings and hypothesis are schematically depicted.

Although we have hypothesized that AdoMet levels remain high because of less demand due to neurodegeneration, we do not have proof for that yet. Because of the complexity of factors that are involved in AdoMet metabolism in $\mathrm{AD}$, other factors in the methylation cycle or for example oxidative stress, could be involved and should be investigated before we can validate our statement. Bernardo shows [1] that 16-18-month-old Tg2576 AD mice have higher Hcy levels than wild-type mice. This could have explained the high-AdoMet levels; however, the AdoMet levels in the 8-month-old APP/PS1 mice in our study, should have been increased compared to 8-month-old wild types, and that was not the case. In addition, high Hcy levels are known to inhibit AdoHcy hydrolase, which results in increase in AdoHcy levels [32], and therefore higher AdoHcy levels would have been expected in our APP/PS1 mice compared to wild types which was also not the case.

The finding that AdoMet levels significantly differ only in 15-month-old APP/PS1 mice compared to their wildtype littermates, but not in 8-month-old mice, also indicates that alterations in AdoMet levels are a consequence of $\mathrm{AD}$ pathology rather than a cause. The difference in AdoMet levels between wild type and $\mathrm{AD}$ mice is also found by Fuso et al. [9]. They show a concentration of $158 \mathrm{nmol} / \mathrm{g}$
AdoMet in TgCRND8 mice compared to $52 \mathrm{nmol} / \mathrm{g}$ AdoMet in their control mice. It could be argued that these mice are much younger and should not be compared with our mice; however, the 3-month-old TgCRND8 animals show early and age-related $\mathrm{A} \beta$ deposition and cognitive impairment what is comparable to our 15-month-old APP/ PS1 mice. In contrast, our 8-month-old APP/PS1 mice show almost no $\mathrm{A} \beta$ plaque deposition and very mild $\operatorname{cog}$ nitive impairment.

The finding that cholesterol- or DHA-containing diets do not alter AdoMet or AdoHcy levels in brain tissue of either wild type or APP/PS1 mice, but in contrast do have effects on AD pathology and cerebral hemodynamics [7, $15,29]$, suggests that cholesterol- or DHA-containing diets do not influence cerebral hemodynamics or AD pathology via the methylation cycle. Thus, AdoMet and AdoHcy are no important key players in the mechanisms explaining the effects of cholesterol and DHA in AD pathology.

\section{Main conclusions}

We measured AdoMet and AdoHcy levels in brain tissue of 8- and 15-month-old wild type and APP/PS1 mice, to investigate whether there is a role for these metabolites of methylation in the $\mathrm{AD}$ brain. The finding that AdoMet levels do not differ between the genotypes in (young) 8month-old mice, but do differ in (older) 15-month-old APP/PS1 mice compared to their wild type littermates,
Fig. 2 Concept model of the hypothetical effects of aging on $S$-adensylmethionine (AdoMet) and $S$-adenosylhomocysteine (AdoHcy) levels in wild type and APP/PS1 mice

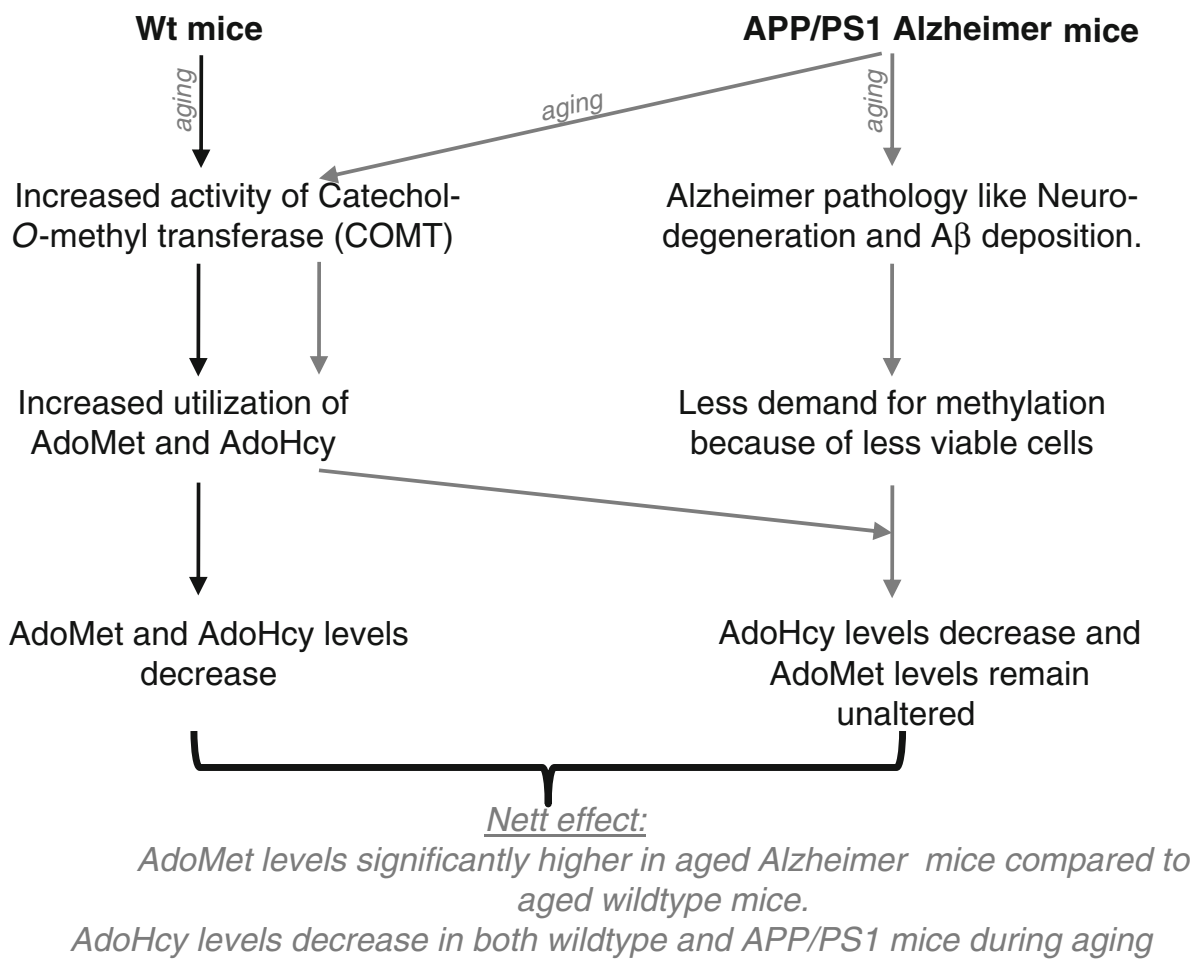


suggests that alterations in AdoMet are a consequence of AD pathology rather than a cause, and it could be suggested that it is the result of neurodegeneration during aging (Fig. 2). However, because of a lack of brain tissue we only measured AdoMet and AdoHcy levels in this experiment. It would, however, be of great value to measure more components of the methylation cycle in future studies to validate our conclusions.

Acknowledgments We would like to thank Henk Arnts for his excellent care giving to our animals. This study is partly supported by an EU 5th framework project (no:QLRT-2002-172), and a Research exchange grant from Zon-MW the Netherlands organization for Health Research and Development (910-31-704).

Open Access This article is distributed under the terms of the Creative Commons Attribution Noncommercial License which permits any noncommercial use, distribution, and reproduction in any medium, provided the original author(s) and source are credited.

\section{References}

1. Bernardo A, McCord M, Troen AM, Allison JD, McDonald MP (2007) Impaired spatial memory in APP-overexpressing mice on a homocysteinemia-inducing diet. Neurobiol Aging 28:11951205

2. Bottiglieri T, Godfrey P, Flynn T, Carney MW, Toone BK, Reynolds EH (1990) Cerebrospinal fluid $S$-adenosylmethionine in depression and dementia: effects of treatment with parenteral and oral $S$-adenosylmethionine. J Neurol Neurosurg Psychiatry 53:1096-1098

3. Bottiglieri T, Hyland K (1994) $S$-adenosylmethionine levels in psychiatric and neurological disorders: a review. Acta Neurol Scand Suppl 154:19-26

4. Collaboration B-VTT (2006) Homocysteine-lowering trials for prevention of cardiovascular events: a review of the design and power of the large randomized trials. Am Heart J 151:282-287

5. Durga J, van Boxtel MP, Schouten EG, Kok FJ, Jolles J, Katan MB, Verhoef P (2007) Effect of 3-year folic acid supplementation on cognitive function in older adults in the FACIT trial: a randomised, double blind, controlled trial. Lancet 369:208-216

6. Finkelstein JD (1990) Methionine metabolism in mammals. J Nutr Biochem 1:228-237

7. Freund-Levi Y, Eriksdotter-Jonhagen M, Cederholm T, Basun $\mathrm{H}$, Faxen-Irving G, Garlind A, Vedin I, Vessby B, Wahlund LO, Palmblad J (2006) Omega-3 fatty acid treatment in 174 patients with mild to moderate Alzheimer disease: OmegAD study: a randomized double-blind trial. Arch Neurol 63:1402-1408

8. Fuso A, Cavallaro RA, Zampelli A, D’Anselmi F, Piscopo P, Confaloni A, Scarpa S (2007) Gamma-secretase is differentially modulated by alterations of homocysteine cycle in neuroblastoma and glioblastoma cells. J Alzheimers Dis 11:275-290

9. Fuso A, Nicolia V, Cavallaro RA, Ricceri L, D’Anselmi F, Coluccia P, Calamandrei G, Scarpa S (2008) B-vitamin deprivation induces hyperhomocysteinemia and brain $S$-adenosylhomocysteine, depletes brain $S$-adenosylmethionine, and enhances PS1 and BACE expression and amyloid-beta deposition in mice. Mol Cell Neurosci 37:731-746

10. Fuso A, Seminara L, Cavallaro RA, D'Anselmi F, Scarpa S (2005) $S$-adenosylmethionine/homocysteine cycle alterations modify DNA methylation status with consequent deregulation of
PS1 and BACE and beta-amyloid production. Mol Cell Neurosci 28:195-204

11. Geleijnse JM, Giltay EJ, Grobbee DE, Donders AR, Kok FJ (2002) Blood pressure response to fish oil supplementation: metaregression analysis of randomized trials. J Hypertens 20:1493-1499

12. Gellekink H, van Oppenraaij-Emmerzaal D, van Rooij A, Struys EA, den Heijer M, Blom HJ (2005) Stable-isotope dilution liquid chromatography-electrospray injection tandem mass spectrometry method for fast, selective measurement of $S$-adenosylmethionine and $S$-adenosylhomocysteine in plasma. Clin Chem 51:1487-1492

13. Goodfellow J, Bellamy MF, Ramsey MW, Jones CJ, Lewis MJ (2000) Dietary supplementation with marine omega- 3 fatty acids improve systemic large artery endothelial function in subjects with hypercholesterolemia. J Am Coll Cardiol 35:265-270

14. Harris WS (1997) n-3 fatty acids and serum lipoproteins: human studies. Am J Clin Nutr 65:1645S-1654S

15. Hooijmans CR, Rutters F, Dederen PJ, Gambarota G, Veltien A, van Groen T, Broersen LM, Lutjohann D, Heerschap A, Tanila H, Kiliaan AJ (2007) Changes in cerebral blood volume and amyloid pathology in aged Alzheimer APP/PS1 mice on a docosahexaenoic acid (DHA) diet or cholesterol enriched Typical Western Diet (TWD). Neurobiol Dis 28:16-29

16. Hooijmans CR, Van der Zee CE, Dederen PJ, Brouwer KM, Reijmer YD, van Groen T, Broersen LM, Lutjohann D, Heerschap A, Kiliaan AJ (2009) DHA and cholesterol containing diets influence Alzheimer-like pathology, cognition and cerebral vasculature in APPswe/PS1dE9 mice. Neurobiol Dis 33:482-498

17. Kerins DM, Koury MJ, Capdevila A, Rana S, Wagner C (2001) Plasma $S$-adenosylhomocysteine is a more sensitive indicator of cardiovascular disease than plasma homocysteine. Am J Clin Nutr 74:723-729

18. Lieber CS, Packer L (2002) S-Adenosylmethionine: molecular, biological, and clinical aspects-an introduction. Am J Clin Nutr 76:1148S-1150S

19. Lonn E, Yusuf S, Arnold MJ, Sheridan P, Pogue J, Micks M, McQueen MJ, Probstfield J, Fodor G, Held C, Genest J Jr (2006) Homocysteine lowering with folic acid and $\mathrm{B}$ vitamins in vascular disease. N Engl J Med 354:1567-1577

20. Luchsinger JA, Tang MX, Miller J, Green R, Mayeux R (2007) Relation of higher folate intake to lower risk of Alzheimer disease in the elderly. Arch Neurol 64:86-92

21. Malouf M, Grimley EJ, Areosa SA (2003) Folic acid with or without vitamin B12 for cognition and dementia. Cochrane Database Syst Rev: CD004514

22. Mato JM, Alvarez L, Ortiz P, Pajares MA (1997) S-adenosylmethionine synthesis: molecular mechanisms and clinical implications. Pharmacol Ther 73:265-280

23. McCully KS (1996) Homocysteine and vascular disease. Nat Med 2:386-389

24. Morrison LD, Smith DD, Kish SJ (1996) Brain S-adenosylmethionine levels are severely decreased in Alzheimer's disease. J Neurochem 67:1328-1331

25. Mulder C, Schoonenboom NS, Jansen EE, Verhoeven NM, van Kamp GJ, Jakobs C, Scheltens P (2005) The transmethylation cycle in the brain of Alzheimer patients. Neurosci Lett 386:6971

26. Okuda N, Ueshima H, Okayama A, Saitoh S, Nakagawa H, Rodriguez BL, Sakata K, Choudhury SR, Curb JD, Stamler J (2005) Relation of long chain n-3 polyunsaturated fatty acid intake to serum high density lipoprotein cholesterol among Japanese men in Japan and Japanese-American men in Hawaii: the INTERLIPID study. Atherosclerosis 178:371-379

27. Peterson GL (1983) Determination of total protein. Methods Enzymol 91:95-119 
28. Ravaglia G, Forti P, Maioli F, Martelli M, Servadei L, Brunetti N, Porcellini E, Licastro F (2005) Homocysteine and folate as risk factors for dementia and Alzheimer disease. Am J Clin Nutr 82:636-643

29. Refolo LM, Malester B, LaFrancois J, Bryant-Thomas T, Wang R, Tint GS, Sambamurti K, Duff K, Pappolla MA (2000) Hypercholesterolemia accelerates the Alzheimer's amyloid pathology in a transgenic mouse model. Neurobiol Dis 7:321-331

30. Scarpa S, Fuso A, D'Anselmi F, Cavallaro RA (2003) Presenilin 1 gene silencing by $S$-adenosylmethionine: a treatment for Alzheimer disease? FEBS Lett 541:145-148
31. Selkoe DJ (2002) Alzheimer's disease is a synaptic failure. Science 298:789-791

32. Selley ML (2007) A metabolic link between $S$-adenosylhomocysteine and polyunsaturated fatty acid metabolism in Alzheimer's disease. Neurobiol Aging 28:1834-1839

33. Stramentinoli G, Gualano M, Catto E, Algeri S (1977) Tissue levels of $S$-adenosylmethionine in aging rats. J Gerontol 32:392394 